\title{
Violence Against the Elderly: Social Representations of Portuguese and Brazilian Caregivers
}

International Workshop on Gerontechnology

IWoG 2018: Gerontechnology pp 295-306 | Cite as

- Felismina Mendes (1) Email author (fm@uevora.pt)View author's OrcID profile (View OrcID profile)

- Tatiana Mestre (1) View author's OrcID profile (View OrcID profile)

- Elaine Santana (2) View author's OrcID profile (View OrcID profile)

- Luciana Reis (2) View author's OrcID profile (View OrcID profile)

- Manuel Lopes (1) View author's OrcID profile (View OrcID profile)

1. Department of Nursing, University of Évora, , Évora, Portugal

2. Postgraduate Programme in Memory: Language and Society, State University of Southwest of Bahia, , Vitória da Conquista, Brazil

Conference paper

First Online: 14 April 2019

- 243 Downloads

Part of the Communications in Computer and Information Science book series (CCIS, volume 1016)

\section{Abstract}

The increase in life expectancy and elderly people in society, coupled with changes in family structure, have highlighted the importance of formal and informal caregivers of elderly people. Objective: To analyse the social representations of violence against the elderly, of two groups of formal and informal caregivers.

Methods: Exploratory, quantitative and qualitative research, supported by the theoretical-methodological reference of the Social Representations Theory and in the context of this, the Central Core Theory. The sample was participated in by 81 formal caregivers from the project "Aging in Safety in the Alentejo Understanding to Act, of the University of Évora" and 20 informal caregivers from the project "Qualification of caregivers and aspects related to the quality of life of the elderly people dependent on primary and tertiary care: Implementation and protocol evaluation, of the State University of Southwest of Bahia”. We used the Free Word Association Technique. The data was analysed by prototypical analysis based on two matrices by the software IRAMUTEQ (Interface de R pour les Analyses Multidimensionnelles de Textes et de Questionnaires) 0.7 alpha 2.

Results: In Portugal, the elements "bad, mistreatment, I will be, sad, anger, 
patience, physical, injustice, irritation and meanness" stood out in the central core. In Brazil the mention of "hitting" was emphasized. Conclusions: In both Portugal and Brazil, physical violence takes on particular significance in the social representations of caregivers, rather than verbal and psychological violence, which is not present in the central core of social representations of violence against the elderly in either of the countries.

\section{Keywords}

Aging Elderly people Caregivers Violence

This is a preview of subscription content, $\underline{\log } \underline{\text { in }}$ to check access.

\section{References}

Abric, J.: Abordagem estrutural das representações sociais: desenvolvimentos recentes. In: Campos, P., Loureiro, M. (eds.) Representações sociais e práticas educativas. UCG, Goiânia (2003a)

Google Scholar (https://scholar.google.com/scholar?

q=Abric\%2C\%2OJ.\%3A\%2oAbordagem\%2oestrutural\%2odas\%2orepresenta\%C3 \%A7\%C3\%B5es\%20sociais\%3A\%20desenvolvimentos\%2orecentes.\%20In\%3A\%2 oCampos\%2C\%2OP.\%2C\%2OLoureiro\%2C\%20M.\%20\%28eds.\%29\%20Represent a\%C3\%A7\%C3\%B5es\%2osociais\%20e\%2opr\%C3\%A1ticas\%2oeducativas.\%2oUC G\%2C\%20Goi\%C3\%A2nia\%20\%282003a\%29)

Abric, J.: La recherche du noyau central et de la zone muette des représentations sociales. In: Abric, J. (ed.) Méthodes d'étude des représentations sociales. Érès, Ramonville-Saint Agne (2003b)

Google Scholar (https://scholar.google.com/scholar?

q=Abric\%2C\%20J.\%3A\%20La\%2orecherche\%2odu\%2onoyau\%2ocentral\%2oet \%2ode\%2ola\%2Ozone\%2omuette\%2odes\%2orepr\%C3\%A9sentations\%20sociale s.\%20In\%3A\%2oAbric\%2C\%20J.\%20\%28ed.\%29\%2oM\%C3\%A9thodes\%2od\%E 2\%80\%99\%C3\%A9tude\%20des\%2orepr\%C3\%A9sentations\%20sociales.\%20\%C3 \%89r\%C3\%A8s\%2C\%2oRamonville-Saint\%20Agne\%20\%282003b\%29)

Apratto, P.: A violência doméstica contra idosos nas áreas de abrangência do Programa Saúde da Família de Niterói (RJ, Brasil). Ciênc. Saúde Coletiva 15(6), 2983-2995 (2010)

CrossRef (https://doi.org/10.1590/S1413-81232010000600037)

Google Scholar (http://scholar.google.com/scholar_lookup?

title=A\%20viol\%C3\%AAncia\%20dom\%C3\%A9stica\%2ocontra\%2oidosos\%2onas \%20\%C3\%A1reas\%2ode\%20abrang\%C3\%AAncia\%2odo\%2oPrograma\%2oSa\%C 3\%BAde\%2Oda\%20Fam\%C3\%ADlia\%2ode\%2oNiter\%C3\%B3i\%20\%28RJ\%2C\% 2oBrasil\%29\&author=P.\%20Apratto\&journal=Ci\%C3\%AAnc.\%20Sa\%C3\%BAde \%20Coletiva\&volume $=15 \&$ issue $=6 \&$ pages $=2983-2995 \&$ publication_year=2010 $)$

Associação Portuguesa de Apoio à Vítima.: Manual Títono - Apoio a Pessoas Idosas Vítimas de Crime e de Violência (2010).

http://www.apav.pt/pdf/Titono_PT.pdf (http://www.apav.pt/pdf/Titono_PT.pdf). Accessed 8 Oct 2018

Ayres, J., Calazams, G., Saletti Filho, H., França Júnior, I.: Risco, vulnerabilidade 
e práticas de prevenção e promoção da saúde. In: Akerman, M., Campos, G., Carvalho, Y., Drumond Jr., M., Minayo, M. (eds.) Tratado de Saúde Coletiva, 2nd edn., pp. 375-416. Hucitec, São Paulo (2012)

Google Scholar (https://scholar.google.com/scholar?

q=Ayres\%2C\%20J.\%2C\%2OCalazams\%2C\%2oG.\%2C\%2oSaletti\%2oFilho\%2C\%2 oH.\%2C\%2oFran\%C3\%A7a\%20J\%C3\%BAnior\%2C\%2OI.\%3A\%2oRisco\%2C\%20 vulnerabilidade\%2oe\%2opr\%C3\%A1ticas\%2ode\%2opreven\%C3\%A7\%C3\%A30\% 20e\%2opromo\%C3\%A7\%C3\%A30\%20da\%20sa\%C3\%BAde.\%20In\%3A\%20Aker man\%2C\%2oM.\%2C\%20Campos\%2C\%2OG.\%2C\%2OCarvalho\%2C\%2OY.\%2C\%2 oDrumond\%2OJr.\%2C\%20M.\%2C\%20Minayo\%2C\%2oM.\%20\%28eds.\%29\%20T ratado\%20de\%20Sa\%C3\%BAde\%2oColetiva\%2C\%2O2nd\%2Oedn.\%2C\%2opp.\%2 0375\%E2\%80\%93416.\%20Hucitec\%2C\%20S\%C3\%A30\%20Paulo\%20\%282012\% 29)

Barbosa, K., Costa, K., Pontes, M., Batista, P., Oliveira, F., Fernandes, M.:

Envelhecimento e vulnerabilidade individual: um panorama dos idosos vinculados à estratégia saúde da família. Texto Contexto Enferm. 26(2), e2700015 (2017)

CrossRef (https://doi.org/10.1590/0104-07072017002700015)

Google Scholar (http://scholar.google.com/scholar_lookup?

title=Envelhecimento\%20e\%20vulnerabilidade\%2oindividual\%3A\%20um\%2opa norama\%20dos\%20idosos\%2ovinculados\%20\%C3\%Ao\%2oestrat\%C3\%A9gia\%2 osa\%C3\%BAde\%20da\%2ofam\%C3\%ADlia\&author=K.\%20Barbosa\&author=K.\% 20Costa\&author=M.\%20Pontes\&author $=$ P.\%2oBatista\&author $=$ F.\%2oOliveira\&a uthor $=$ M.\%2oFernandes\&journal=Texto\%20Contexto\%20Enferm. $\&$ volume $=26 \&$ issue $=2$ \&pages $=$ e2700015\&publication_year $=2017)$

Batista, M., Almeida, M., Lancman, S.: Cuidadores formais de idosos: contextualização histórica no cenário brasileiro. Rev. Bras. Geriatr. Gerontol. 17(4), 879-885 (2014) CrossRef (https://doi.org/10.1590/1809-9823.2014.13148) Google Scholar (http://scholar.google.com/scholar_lookup? title=Cuidadores\%2oformais\%2ode\%2oidosos\%3A\%2ocontextualiza\%C3\%A7\%C 3\%A30\%2ohist\%C3\%B3rica\%20no\%2ocen\%C3\%A1rio\%2obrasileiro\&author=M. \%20Batista\&author=M.\%20Almeida\&author=S.\%2OLancman\&journal=Rev.\%2O Bras.\%20Geriatr.\%20Gerontol.\&volume $=17 \&$ issue $=4 \&$ pages $=879-$ 885\&publication_year=2014)

Brito, A., Belloni, E., Castro, A., Camargo, B., Giacomozzi, A.: Representações sociais do cuidado e da velhice no Brasil e Itália. Psic. Teor. Pesq. 34, 3455 (2018) Google Scholar (http://scholar.google.com/scholar_lookup? title=Representa\%C3\%A7\%C3\%B5es\%20sociais\%20do\%20cuidado\%20e\%2oda\% 20velhice\%20no\%20Brasil\%20e\%20It\%C3\%A1lia\&author=A.\%2oBrito\&author= E.\%20Belloni\&author=A.\%20Castro\&author=B.\%20Camargo\&author=A.\%20Gia comozzi\&journal=Psic.\%20Teor.\%20Pesq.\&volume=34\&pages $=3455 \&$ publicatio n_year=2018)

Camargo, B., Justo, A.: IRAMUTEQ: um software gratuito para análise de dados textuais. Temas Psicol. 21(2), 513-518 (2013)

CrossRef (https://doi.org/10.9788/TP2013.2-16) Google Scholar (http://scholar.google.com/scholar_lookup? title=IRAMUTEQ\%3A\%20um\%20software\%2ogratuito\%2opara\%20an\%C3\%A1li se\%20de\%20dados\%20textuais\&author=B.\%20Camargo\&author=A.\%20Justo\&j 
ournal=Temas $\% 20$ Psicol. $\&$ volume $=21 \&$ issue $=2 \&$ pages $=513^{-}$

518\&publication_year $=2013$ )

Castro, A., Guilam, M., Sousa, E., Marcondes, W.: Violência na velhice:

abordagens em periódicos nacionais indexados. Ciênc. Saúde Coletiva 18(5), 1283-1292 (2013)

CrossRef (https://doi.org/10.1590/S1413-81232013000500013)

Google Scholar (http://scholar.google.com/scholar_lookup?

title=Viol\%C3\%AAncia\%20na\%20velhice\%3A\%20abordagens\%20em\%2operi\%C 3\%B3dicos\%2onacionais\%20indexados\&author=A.\%20Castro\&author=M.\%20G uilam\&author $=$ E.\%20Sousa\&author $=$ W.\%2oMarcondes\&journal=Ci\%C3\%AAnc. \%20Sa\%C3\%BAde\%2oColetiva\&volume=18\&issue =5\&pages =12831292\&publication_year=2013)

Danic, I.: La notion de représentation pour les sociologues. Premier aperçu (2006)

Google Scholar (https://scholar.google.com/scholar? q=Danic\%2C\%20I.\%3A\%20La\%20notion\%20de\%20repr\%C3\%A9sentation\%20p our\%2oles\%20sociologues.\%2oPremier\%2oaper\%C3\%A7u\%20\%282006\%29)

Davison, E., McCabe, M., Visser, S., Hudgson, C., Buchanan, G., George, K.:

Controlled trial of dementia training with a peer support group for aged care staff. Int. J. Geriatr. Psychiatry 22, 868-873 (2007)

CrossRef (https://doi.org/10.1002/gps.1754)

Google Scholar (http://scholar.google.com/scholar_lookup?

title=Controlled\%20trial\%200f\%2odementia\%2otraining\%20with\%20a\%2opeer \%20support\%2ogroup\%2ofor\%20aged\%2ocare\%2ostaff\&author=E.\%2oDavison \&author=M.\%20McCabe\&author=S.\%20Visser\&author=C.\%20Hudgson\&author =G.\%20Buchanan\&author=K.\%20George\&journal=Int.\%20J.\%20Geriatr.\%20Ps ychiatry\&volume=22\&pages $=868-873 \&$ publication_year $=2007$ )

Di Giacomo, J.: Aspects méthodologiques de l'analyse des réprésentations sociales. Cah. Psychol. Cogn. Curr. Psychol. Cogn. 1, 397-422 (1981)

Google Scholar (http://scholar.google.com/scholar_lookup?

title=Aspects\%20m\%C3\%Agthodologiques\%20de\%201\%E2\%80\%99analyse\%2od es\%20r\%C3\%A9pr\%C3\%A9sentations\%20sociales\&author=J.\%20Giacomo\&jour nal=Cah.\%20Psychol.\%20Cogn.\%20Curr.\%20Psychol.\%20Cogn.\&volume=1\&pag es=397-422\&publication_year=1981)

Dias, I.: Violência doméstica e justiça: respostas e desafios. Soc. Rev. Fac. Let.

Univ. Porto 2o, 245-262 (2010)

Google Scholar (http://scholar.google.com/scholar_lookup?

title=Viol\%C3\%AAncia\%20dom\%C3\%A9stica\%20e\%20justi\%C3\%A7a\%3A\%20re spostas\%20e\%2odesafios\&author=I.\%20Dias\&journal=Soc.\%20Rev.\%20Fac.\%2 oLet.\%20Univ.\%20Porto\&volume=20\&pages=245-262\&publication_year=2010)

Duarte, N., Barbosa, C.: Manual do Envelhecimento Ativo. In: Ribeiro, O., Paúl, C. (eds.) Envelhecimento Ativo, 1st edn. Lidel, Lisboa (2011)

Google Scholar (http://scholar.google.com/scholar_lookup?

title=Manual\%2odo\%2oEnvelhecimento\%20Ativo\&author=N.\%2oDuarte\&autho $\mathrm{r}=\mathrm{C} . \% 20$ Barbosa\&publication_year $=2011$ )

Instituto Brasileiro de Geografia e Estatística. Pesquisa Nacional por Amostra de Domicílios: síntese de indicadores 2013. Coordenação de Trabalho e Rendimento, Rio de Janeiro, 2 edn., 296 p. (2015) 
Google Scholar (https://scholar.google.com/scholar?

q=Instituto\%20 Brasileiro\%20de\%20Geografia\%20e\%20Estat\%C3\%ADstica.\%20 Pesquisa\%20Nacional\%20por\%20Amostra\%20de\%2oDomic\%C3\%ADlios\%3A\%2 os\%C3\%ADntese\%2ode\%2oindicadores\%202013.\%20Coordena\% 3 3\%A7\%C3\%A 30\%20de\%20Trabalho\%20e\%2oRendimento\%2C\%20Rio\%2Ode\%2OJaneiro\%2C \%202\%20edn.\%2C\%20296\%20p.\%20\%282015\%29)

Junqueira, L.: A noção de representação social na sociologia contemporânea.

Estudos Soc. 18(19), 145-161 (2005)

Google Scholar (http://scholar.google.com/scholar_lookup? title=A\%20no\%C3\%A7\%C3\%A30\%2ode\%2orepresenta\%C3\%A7\%C3\%A30\%20so cial\%20na\%20sociologia\%20contempor\%C3\%A2nea\&author=L.\%20Junqueira\&j ournal=Estudos $\% 20$ Soc.$\&$ volume $=18 \&$ issue $=19$ \&pages $=145^{-}$

161\&publication_year=2005)

Leite, M., Hildebrandt, L., dos Santos, A.: Maus-tratos a idosos no domicílio: concepção de familiares. Rev. Bras. Geriatr. Gerontol. 11(2), 209-221 (2008) CrossRef (https://doi.org/10.1590/1809-9823.2008.11027) Google Scholar (http://scholar.google.com/scholar_lookup?title=Maustratos\%20a\%20idosos\%20no\%20domic\%C3\%ADlio\%3A\%20concep\%C3\%A7\%C3 \%A30\%20de\%20familiares\&author=M.\%20Leite\&author=L.\%20Hildebrandt\&au thor=A.\%20Santos\&journal=Rev.\%20Bras.\%20Geriatr.\%20Gerontol.\&volume=11 \&issue $=2 \&$ pages $=209-221 \&$ publication_year $=2008$ )

Luoma, M., et al.: Prevalence study of abuse and violence against older women: Results of a multicultural survey conducted in Austria, Belgium, Finland, Lithuania and Portugal. THL, Department of Health and Welfare, Helsinki, Finland (2011)

Google Scholar (https://scholar.google.com/scholar? q=Luoma\%2C\%2oM.\%2C\%2oet\%20al.\%3A\%20Prevalence\%2ostudy\%20of\%20a buse\%20and\%20violence\%20against\%20older\%2Owomen\%3A\%2oResults\%20of \%20a\%20multicultural\%20survey\%20conducted\%20in\%20Austria\%2C\%2oBelgi um\%2C\%2oFinland\%2C\%2oLithuania\%20and\%2oPortugal.\%20THL\%2C\%2oDe partment\%20of\%20Health\%2Oand\%20Welfare\%2C\%20Helsinki\%2C\%2oFinland \%20\%282011\%29)

Mariano, P., Baldissera, V., Martins, J., Carreira, L.: Nursing work organization in long-stay institutions for the elderly: relationship to pleasure and suffering at work. Texto Contexto Enferm. 24(3), 756-765 (2015) CrossRef (https://doi.org/10.1590/0104-070720150-1150014) Google Scholar (http://scholar.google.com/scholar_lookup? title=Nursing\%20work\%20organization\%20in\%2olongstay\%2oinstitutions\%2ofor\%2othe\%2oelderly\%3A\%2orelationship\%20to\%2ople asure\%20and\%20suffering\%20at\%20work\&author=P.\%20Mariano\&author=V.\% 2oBaldissera\&author=J.\%20Martins\&author=L.\%20Carreira\&journal=Texto\%20 Contexto\%20Enferm.$\&$ volume $=24 \&$ issue $=3 \&$ pages $=756-$ 765\&publication_year=2015)

Observatório Português dos Sistemas de Saúde. Acesso aos cuidados de saúde. Um direito em risco? Relatório de Primavera (2015). http://www.opss.pt/sites/opss.pt/files/RelatorioPrimavera2015.pdf (http://www.opss.pt/sites/opss.pt/files/RelatorioPrimavera2015.pdf)

Oliveira, M., Gomes, A., Amaral, C., Santos, L.: Características dos idosos vítimas 
de violência doméstica no Distrito Federal. Rev. Bras. Geriatr. Gerontol. 15(3), $555-566$ (2012)

CrossRef (https://doi.org/10.1590/S1809-98232012000300016)

Google Scholar (http://scholar.google.com/scholar_lookup?

title=Caracter\%C3\%ADsticas\%20dos\%20idosos\%20v\%C3\%ADtimas\%2ode\%2ovi ol\%C3\%AAncia\%20dom\%C3\%A9stica\%20no\%20Distrito\%2oFederal\&author=M. \%20Oliveira\&author=A.\%20Gomes\&author=C.\%20Amaral\&author $=$ L.\%20Santo s\&journal=Rev.\%20Bras.\%20Geriatr.\%20Gerontol.\&volume=15\&issue=3\&pages $=555-566 \&$ publication_year $=2012$ )

Organização Mundial De Saúde: Relatório Mundial sobre a Prevenção da Violência 2014. Núcleo de Estudos da Violência (Trad.), São Paulo (2014) Google Scholar (https://scholar.google.com/scholar? q=Organiza\%C3\%A7\%C3\%A30\%20Mundial\%20De\%20Sa\%C3\%BAde\%3A\%2oRe lat\%C3\%B3rio\%2oMundial\%20sobre\%20a\%20Preven\%C3\%A7\%C3\%A30\%20da \%20Viol\%C3\%AAncia\%202014.\%20N\%C3\%BAcleo\%20de\%20Estudos\%20da\%2 oViol\%C3\%AAncia\%20\%28Trad.\%29\%2C\%20S\%C3\%A30\%20Paulo\%20\%28201 4\%29)

PORDATA Fundação Francisco Manuel dos Santos, Esperança de vida à nascença: total e por sexo. Portugal. Europa. Municípios.

https://www.pordata.pt/Portugal/Esperan\%C3\%A7a+de+vida+\%C3\%Ao+nascen\%C3\%A7a+total+e+por+sexo+ (base+tri\%C3\%A9nio+a+partir+de+2001)-418

(https://www.pordata.pt/Portugal/Esperan\%25C3\%25A7a\%2bde\%2bvida\%2b\% 25C3\%25Ao\%2bnascen\%25C3\%25A7a\%2btotal\%2be\%2bpor\%2bsexo\%2b(base\% 2btri\%25C3\%25A9nio\%2ba\%2bpartir\%2bde\%2b2001)-418). Accessed 8 Oct 2018

PORDATA, Fundação Francisco Manuel dos Santos, Indicadores de Envelhecimento.

https://www.pordata.pt/Portugal/Indicadores+de+envelhecimento-526

(https://www.pordata.pt/Portugal/Indicadores\%2bde\%2benvelhecimento-526). Accessed 8 Oct 2018

PORDATA, Fundação Francisco Manuel dos Santos, Indicadores de Envelhecimento. https://www.pordata.pt/Municipios/\%C3\%8Dndice+de+envelhecimento-458 (https://www.pordata.pt/Municipios/\%25C3\%258Dndice\%2bde\%2benvelhecim ento-458). Accessed 8 Oct 2018

Porto, M.: Crenças, valores e representações sociais da violência. Sociologias 8(16), 250-273 (2006)

CrossRef (https://doi.org/10.1590/S1517-45222006000200010) Google Scholar (http://scholar.google.com/scholar_lookup? title=Cren\%C3\%A7as\%2C\%2ovalores\%2oe\%2orepresenta\%C3\%A7\%C3\%B5es\%2 osociais\%20da\%20viol\%C3\%AAncia\&author=M.\%20Porto\&journal=Sociologias \&volume=8\&issue=16\&pages=250-273\&publication_year=2006)

Prado, S., Sayd, J.: A gerontologia como campo do conhecimento científico: conceito, interesses e projeto político. Cien. Saude Colet. 11(2), 91-501 (2006) CrossRef (https://doi.org/10.1590/S1413-81232006000200026)

Google Scholar (http://scholar.google.com/scholar_lookup? title=A\%20gerontologia\%20como\%20campo\%20do\%20conhecimento\%20cient\% C3\%ADfico\%3A\%2Oconceito\%2C\%2ointeresses\%20e\%2oprojeto\%2opol\%C3\%A Dtico\&author=S.\%2OPrado\&author=J.\%2OSayd\&journal=Cien.\%20Saude\%2oCo 
let.$\&$ volume $=11 \&$ issue $=2 \&$ pages $=91-501 \&$ publication_year $=2006)$

Ratinaud, P.: IRAMUTEQ: Interface de R pour les Analyses Multidimensionnelles de Textes et de Questionnaires [Computer software] (2009).

http://www.iramuteq.org (http://www.iramuteq.org). Accessed 8 Oct 2018

Sá, C.: Núcleo Central das Representações Sociais, 2nd edn. Vozes, Petrópolis (1996)

Google Scholar (http://scholar.google.com/scholar_lookup?

title=N\%C3\%BAcleo\%20Central\%20das\%2oRepresenta\%C3\%A7\%C3\%B5es\%20

Sociais\&author=C.\%20S\%C3\%A1\&publication_year=1996)

Silveira, E., Vieira, L., Souza, J.: Elevada prevalência de obesidade abdominal em idosos e associação com diabetes, hipertensão e doenças respiratórias. Ciênc.

Saúde Coletiva 23(3), 903-912 (2018)

CrossRef (https://doi.org/10.1590/1413-81232018233.01612016)

Google Scholar (http://scholar.google.com/scholar_lookup?

title=Elevada\%2opreval\%C3\%AAncia\%20de\%20obesidade\%20abdominal\%2oem \%2oidosos\%20e\%2oassocia\%C3\%A7\%C3\%A30\%20com\%2odiabetes\%2C\%2ohip ertens\% 3 3\%A30\%20e\%2odoen\%C3\%A7as\%2orespirat\%C3\%B3rias\&author=E.\% 20Silveira\&author $=$ L.\%20Vieira\&author $=$ J.\%20Souza\&journal=Ci\%C3\%AAnc.\% 20Sa\%C3\%BAde\%2oColetiva\&volume=23\&issue $=3$ \&pages $=903-$

912\&publication_year=2018)

Torres, T., Camargo, B.: Redes associativas e representações sociais do envelhecimento e rejuvenescimento para diferentes grupos etários. In: Lopes, M., Mendes, F., Silva, A. (eds.) Envelhecimento: Estudos e Perspetivas. Martinari, São Paulo (2014)

Google Scholar (https://scholar.google.com/scholar?

q=Torres\%2C\%20T.\%2C\%20Camargo\%2C\%20B.\%3A\%2oRedes\%2oassociativas \%20e\%2orepresenta\%C3\%A7\%C3\%B5es\%2osociais\%20do\%2oenvelhecimento\% 20e\%2orejuvenescimento\%20para\%20diferentes\%2ogrupos\%2oet\%C3\%A1rios. \%20In\%3A\%2OLopes\%2C\%2OM.\%2C\%2OMendes\%2C\%2OF.\%2C\%2oSilva\%2C\% 20A.\%20\%28eds.\%29\%20Envelhecimento\%3A\%20Estudos\%20e\%2oPerspetivas \%20Martinari\%2C\%20S\%C3\%A30\%20Paulo\%20\%282014\%29)

Veras, R., Oliveira, M.: Envelhecer no Brasil: a construção de um modelo de cuidado. Ciênc. Saúde Coletiva 23(6), 1929-1936 (2018)

CrossRef (https://doi.org/10.1590/1413-81232018236.04722018)

Google Scholar (http://scholar.google.com/scholar_lookup?

title=Envelhecer\%20no\%20Brasil\%3A\%20a\%20constru\%C3\%A7\%C3\%A30\%20d e\%20um\%20modelo\%20de\%20cuidado\&author=R.\%20Veras\&author=M.\%20Ol iveira\&journal=Ci\%C3\%AAnc.\%20Sa\%C3\%BAde\%2oColetiva\&volume=23\&issue $=6 \&$ pages $=1929-1936 \&$ publication_year $=2018$ )

Vieira, C., Fialho, A., Freitas, C., Jorge, M.: Práticas do cuidador informal do idoso no domicílio. Rev. Bras. Enferm. 64(3), 570-579 (2011)

CrossRef (https://doi.org/10.1590/Soo34-71672011000300023)

Google Scholar (http://scholar.google.com/scholar_lookup?

title=Pr\%C3\%A1ticas\%2odo\%2ocuidador\%2oinformal\%2odo\%2oidoso\%20no\%2 odomic\%C3\%ADlio\&author $=\mathrm{C} . \% 20$ Vieira\&author=A.\%20Fialho\&author $=\mathrm{C} . \% 20$ Freitas\&author=M.\%20Jorge\&journal=Rev.\%20Bras.\%20Enferm.\&volume=64\&i ssue $=3 \&$ pages $=570-579 \&$ publication_year $=2011)$

World Health Organization: Informe mundial sobre la violencia y la salud (2002) 


\section{Copyright information}

(C) Springer Nature Switzerland AG 2019

\section{About this paper}

Cite this paper as:

Mendes F., Mestre T., Santana E., Reis L., Lopes M. (2019) Violence Against the Elderly: Social Representations of Portuguese and Brazilian Caregivers. In: García-Alonso J., Fonseca C. (eds) Gerontechnology. IWoG 2018. Communications in Computer and Information Science, vol 1016. Springer, Cham

- First Online 14 April 2019

- DOI https://doi.org/10.1007/978-3-030-16028-9_26

- Publisher Name Springer, Cham

- Print ISBN 978-3-030-16027-2

- Online ISBN 978-3-030-16028-9

- eBook Packages Computer Science

- Buythis book on publisher's site

- $\underline{\text { Reprints and Permissions }}$

\section{Personalised recommendations}

\section{SPRINGER NATURE}

(C) 2019 Springer Nature Switzerland AG. Part of $\underline{\text { pringer Nature. }}$

Not logged in Not affiliated 85.138.7.232 